Original clinical investigation

Open Access

\title{
Resistance to aspirin is increased by ST-elevation myocardial infarction and correlates with adenosine diphosphate levels Catharina Borna ${ }^{1}$, Eduardo Lazarowski ${ }^{2}$, Catharina van Heusden ${ }^{2}$, Hans Öhlin ${ }^{1}$ and David Erlinge*1
}

\begin{abstract}
Address: ${ }^{1}$ Department of Cardiology, Heart \& Lung Division, Lund University Hospital, Sweden and ${ }^{2}$ Department of Medicine, University of North Carolina, School of Medicine, Chapel Hill, USA

Email: Catharina Borna - catharina.borna@skane.se; Eduardo Lazarowski - eduardo_lazarowski@med.unc.edu; Catharina van Heusden - c_vanheusden@med.unc.edu; Hans Öhlin - hans.ohlin@skane.se; David Erlinge* - david.erlinge@med.lu.se

* Corresponding author
\end{abstract}

Published: 26 July 2005

Thrombosis Journal 2005, 3:10 doi:10.1 186/1477-9560-3-10
Received: 06 June 2005

Accepted: 26 July 2005

This article is available from: http://www.thrombosisjournal.com/content/3/I/10

(C) 2005 Borna et al; licensee BioMed Central Ltd.

This is an Open Access article distributed under the terms of the Creative Commons Attribution License (http://creativecommons.org/licenses/by/2.0), which permits unrestricted use, distribution, and reproduction in any medium, provided the original work is properly cited.

\begin{abstract}
Background: To be fully activated platelets are dependent on two positive feedback loops; the formation of thromboxane $A_{2}$ by cyclooxygenase in the platelets and the release of ADP. We wanted to evaluate the effect of aspirin on platelet function in patients with acute coronary syndromes and we hypothesized that increased levels of ADP in patients with acute coronary syndromes could contribute to aspirin resistance.
\end{abstract}

Methods: Platelet activity in 135 patients admitted for chest pain was assessed with PFA-100. An epinephrine-collagen cartridge (EPI-COLL) was used for the detection of aspirin resistance together with an ADP-collagen cartridge (ADP-COLL). ADP was measured with hplc from antecubital vein samples. Three subgroups were compared: chest pain with no sign of cardiac disease (NCD), NonST-elevation myocardial infarction (NSTEMI) and STEMI.

Results: Platelet activation was increased for the STEMI group compared NCD. Aspirin resistance defined as $<193 \mathrm{sec}$ in EPI-COLL was $9.7 \%$ in NCD, and increased to $26.0 \%$ (n.s.) in NSTEMI and $83.3 \%(\mathrm{p}<0.00 \mathrm{I})$ in STEMI. Chronic aspirin treatment significantly reduced platelet aggregation in NCD and NSTEMI, but it had no effect in STEMI. Plasma levels of ADP were markedly increased in STEMI ( $905 \pm 72 \mathrm{I} \mathrm{nmol} / \mathrm{l}, \mathrm{p}<0.0 \mathrm{l})$, but not in NSTEMI $(3 \mathrm{I} 7 \pm 245)$, compared to NCD (334 \pm 27 , mean $\pm S D$ ). ADP levels correlated with increased platelet activity measured with ADP-COLL $(r=-0.30, p<0.05)$. Aspirin resistant patients (EPI-COLL $<193 \mathrm{sec})$ had higher ADP levels compared to aspirin responders (734 \pm 807 vs. $282 \pm 187$ nmol/l, mean $\pm S D, p<0.05)$.

Conclusion: Platelets are activated and aspirin resistance is more frequent in STEMI, probably due to a general activation of platelets. ADP levels are increased in STEMI and correlates with platelet activation. Increased levels of ADP could be one reason for increased platelet activity and aspirin resistance. 


\section{Background}

To be fully activated platelets are dependent on two positive feedback loops; the formation of thromboxane $A_{2}$ by cyclooxygenase in the platelets and the release of ADP from dense platelet granules. Thromboxane $\mathrm{A}_{2}$ and ADP then activates specific receptors on the extracellular side of the platelet membrane. Therapeutic intervention aimed at the first positive feedback loop by inhibiting cyclooxygenase with aspirin is highly efficient in reducing death and cardiovascular events by approximately $25 \%$ [1]. However, ADP may be even more important as evidenced by the CAPRIE-study, in which the ADP receptor antagonist clopidogrel was more beneficial than aspirin in reducing cardiovascular events [2]. Furthermore, the CURE and CREDO studies have established clopidogrel in combination with aspirin as a valuable treatment for acute coronary syndromes $[3,4]$.

The platelet inhibitory effect of aspirin varies and aspirin resistance has been found in $9-45 \%$ of patients [5-7]. Little is known about the clinical consequences of aspirin resistance but lately two different studies indicate that aspirin resistance could be associated with an increased number of cardiovascular events [8,9].

Platelet activation is difficult to assess. Laboratory tests available are either not sufficiently reliable or rather complicated and therefore ineligible for clinical routine use. In this study we used a novel platelet function test, PFA- 100. PFA-100 is an ex vivo assay of shear stress induced platelet adhesion and aggregation in whole blood. It simulates an injured blood vessel by a collagen-coated membrane together with either epinephrine or ADP. It has been found to be a sensitive test of aspirin resistance [6]. Aspirin resistance has previously been studied in healthy controls and in stable patients with a previous myocardial infarction.

In this study we wanted to evaluate the effect of aspirin on platelet function in patients with acute coronary syndromes. Furthermore, we hypothesized that increased levels of ADP in patients with acute coronary syndromes could contribute to aspirin resistance.

\section{Methods \\ Patients}

135 patients were enrolled from patients admitted for chest pain to the emergency ward, Lund University. Hospital between 2001-2003. Patients with chest pain within the last hour before admittance were eligible for inclusion. Patients were defined as aspirin users or patients not using aspirin the last three weeks (and not receiving aspirin during transport to hospital). The use of aspirin was defined as daily intake of aspirin for at least one week before admittance. Most patients were on aspirin $75 \mathrm{mg}$ once daily, but a few $(<10 \%)$ were on $320 \mathrm{mg}$ once daily. Exclusion criteria were: ingestion of clopidogrel, dipyridamole, nonsteroidal antiinflammatory drugs, heparin, low molecular heparin, warfarin, receiving bolus dose of aspirin on their way to hospital, platelet count $<140 \times 10^{9} / \mathrm{l}$, hemoglobin $<90 \mathrm{~g} / \mathrm{l}$, renal failure (creatinine $>140 \mu \mathrm{mol} /$ 1) or hemolysis in blood sample. The Human Ethics Committee of Lund University approved the project. All participants gave informed written consent before enrolment.

Based on the diagnosis at discharge, three prespecified subgroups were compared: chest pain with no sign of cardiac disease (NCD), Non ST-elevation myocardial infarction (NSTEMI) and STEMI. The NCD group presented no recent ECG changes and normal values of TNT. Exercise test where appropriate before discharge were negative. A minor number of patients in the NSTEMI and STEMI group were on beta-blocker, ACE-inhibitors and Ca-channel blockers. However, the groups were to small for subgroup analysis.

\section{PFA-100 system testing}

The PFA-100 system has been described in detail by Kundu and co-workers [10]. The PFA-100 uses a disposable test cartridge that simulates an injured blood vessel. The PFA-100 simulates primary haemostasis by flowing whole blood at a high shear rate through an aperture (147 $\mu \mathrm{m}$ diameter) cut into a collagen-coated membrane coated with either ADP $(50 \mu \mathrm{g})$ or epinephrine $(10 \mu \mathrm{g})$, where it comes into contact with the membrane surface and aggregate. A platelet plug forms, with occlusion of the aperture and cessation of blood flow. The closure time reflects platelet function in the sample evaluated. Shorter closure times indicate increased platelet aggregation. Testing was done in whole blood from antecubital vein samples anticoagulated with $3.8 \%$ sodium citrate. Samples were obtained at admission in 135 patients. PFA-100 tests were performed within $30 \mathrm{~min}$ after blood sampling, within 1 hour after admission to hospital. The epinephrine-collagen cartridge (EPI-COLL) is sensitive to aspirin and can be used for the detection of aspirin resistance [6]. The ADP-collagen cartridge (ADP-COLL) is only weakly sensitive to aspirin. Aspirin resistance was defined as normal EPI-COLL closure times $(<193 \mathrm{sec})$ based on the $90 \%$ central interval in a normal population [6].

\section{Nucleotide measurements}

Nucleotides were measured in a total of 64 patients $(16$ patients with STEMI, 16 patients with NSTEMI and 32 patients with NCD). ADP was measured with hplc from antecubital vein samples. Sampling was done at admission. $5 \mathrm{ml}$ blood was added to tubes containing citrate and immediately centrifuged for $10 \mathrm{~min}$ at $1200 \mathrm{G}, 4^{\circ} \mathrm{C}$. Platelet contamination was excluded by Burker chamber examination. The plasma was aspirated and mixed with 
an equal amount of $10 \%$ TCA to precipitate all proteins and inactivate ectonucleotideases. After centrifugation the protein free supernatant was frozen at $-80^{\circ}$. Samples were sent on dry ice by courier, to Department of Medicine, University of North Carolina, School of Medicine, Chapel Hill, USA for analysis.

Samples were thawed at room temperature and TCA was extracted three times with six volumes of ethyl ether. Ethyl ether was removed by gassing $\mathrm{N}_{2}$, and the resulting samples diluted in the corresponding nucleotide assay buffer as indicated below.

\section{Luciferin-luciferase assay}

This assay has been previously described in detail elsewhere [11]. Typically, extracts were diluted 1:20 in HEPES-buffered Hanks Balanced Salt Solution (HBSS, pH. 7.4) and a 30- $\mu$ l sample was added to a test tube and the volume adjusted to $300 \mu \mathrm{l}$ with HPLC-grade $\mathrm{H}_{2} \mathrm{O}$. The luciferin-luciferase reaction mix $(100 \mu \mathrm{l})$ was added to tubes with a built-in injector into the light protected chamber of an Auto-Lumat LB953 luminometer. Luminescence was subsequently recorded during 10 seconds and compared against an ATP standard curve performed in parallel. Luminescence was linear with slope of one between 0.1 and $1000 \mathrm{nM}$ ATP.

\section{Derivatization of adenosine and adenine nucleotides}

We have adopted and slightly modified a derivatization protocol originally described by Levitt and co-workers [12]. Sample extracts were incubated for $30 \mathrm{~min}$ at $72^{\circ} \mathrm{C}$ in the presence of $1.0 \mathrm{M}$ chloroacetaldehyde and $25 \mathrm{mM}$ $\mathrm{Na}_{2} \mathrm{HPO}_{4}$ (pH 4.0) in a 200- $\mu$ l final volume. Samples were transferred to ice, alkalinized with $50 \mu \mathrm{l} 0.5 \mathrm{M}$ $\mathrm{NH}_{4} \mathrm{HCO}_{3}$, and analyzed by HPLC within $24 \mathrm{~h}$. Identification and quantification of ethenylated species were performed with an automated Waters HPLC apparatus equipped with a fluorescence detector. Derivatized samples were transferred to $0.7 \mathrm{ml}$ plastic shell vials and kept at $4{ }^{\circ} \mathrm{C}$ in the sample injector rack. A $100 \mu \mathrm{l}$ sample aliquot was injected into a $250-\mathrm{mm}, 10 \mu \mathrm{m}$ Hamilton PRP$\mathrm{X} 100$ anion exchange column. The mobile phase $(2 \mathrm{ml} /$ min, $30 \%$ methanol) developed linearly from 0.250 to $0.275 \mathrm{M} \mathrm{NH}_{4} \mathrm{HCO}_{3}(\mathrm{pH} 8.5)$ during the first $8 \mathrm{~min}$, remaining isocratic at $0.275 \mathrm{M} \mathrm{NH}_{4} \mathrm{HCO}_{3}$ for additional 4 $\mathrm{min}$. The column was subsequently rinsed for $3 \mathrm{~min}$ with $0.425 \mathrm{M} \mathrm{NH}_{4} \mathrm{HCO}_{3}$ in $30 \%$ methanol, and re-equilibrated to the initial conditions for $15 \mathrm{~min}$. Elution times (in min) were: $\varepsilon$-ADO, 3.2; $\varepsilon$-AMP, 5.9; $\varepsilon$-ADP, 7.6, and $\varepsilon$ ATP, 9.4.

\section{Reagents}

$\left[{ }^{14}\right.$ Clglucose-1P (300 mCi/mmol) and molecular biology grade ATP and UTP were purchased from Amersham Pharmacia Biotech (Piscataway, NJ). ADP, AMP, and adenos- ine were from Roche Molecular Biochemicals (Indianapolis, IN). Etheno-adenyl standards were from Sigma (St. Louis, MO). Firefly luciferase and luciferin were purchased from PharMingen International (San Diego, $\mathrm{CA}$ ). Other chemicals were of the highest purity available.

\section{Calculation and statistics}

Calculations and statistics were performed using the GraphPad Prism 3.02 software. Values are presented as mean \pm SD. Statistical significance was accepted when $P<$ 0.05 (two-tailed test). For continuous variables KruskalWallis test followed by Dunnett multiple comparisons test was used. Spearman's rank correlation coefficient test was used for regression analysis. Categorical variables were compared using the Fisher exact test.

\section{Ethics}

The Ethics Committee of Lund University approved the project. The study complies with the Declaration of Helsinki. All patients gave written consent to participate in the study.

\section{Results}

\section{Patient characteristics}

The clinical characteristics of the NCD, NSTEMI and STEMI groups are shown in Table 1.

\section{PFA- 100}

In patients without aspirin therapy there was an increased platelet activation in the STEMI group compared to NCD in the EPI-coll. NCD: $139 \pm 44$, NSTEMI: $121 \pm 23$ (n.s.), STEMI: $99 \pm 28 \mathrm{sec}(\mathrm{p}<0.001$, mean \pm SD). (Fig 1a.) These differences were also seen in patients on aspirin. NCD: $280 \pm 41$, NSTEMI: $243 \pm 72$ (n.s.), STEMI: $116 \pm 56 \mathrm{sec}$ $(\mathrm{p}<0.001$, Fig 2a). Lower values indicate increased platelet activation. Similar results were observed with ADPCOLL measurements (Fig $1 \mathrm{~b}$ and $2 \mathrm{~b}$ ).

Chronic aspirin treatment (at least one week before admission) significantly reduced platelet aggregation in NCD and NSTEMI as seen by increased PFA-100 times for EPI-COLL, but only in NCD for ADP-COLL (Fig 2). However, aspirin had no effect in either EPI-COLL or ADPCOLL in patients with STEMI (Fig 2). Aspirin resistance defined as $<193 \mathrm{sec}$ in EPI-COLL was $9.7 \%$ in NCD, and increased to $26.0 \%$ in NSTEMI (n.s.) and $83.3 \%$ in $\operatorname{STEMI}(\mathrm{p}<0,001)$.

For PFA-100 measurements there were no significant correlation with age, diabetes, hemoglobin, $\mathrm{CKMB}$, troponin $\mathrm{T}$ or platelet levels.

\section{Nucleotide release}

Plasma levels of ADP were markedly increased in STEMI $(905 \pm 721 \mathrm{nmol} / \mathrm{l}, \mathrm{p}<0.01)$, but not in NSTEMI $(317 \pm$ 
245), compared to NCD $(334 \pm 271$, mean \pm SD) (Fig 3$)$. Similar findings were found for other purines (ATP and AMP). ADP levels correlated with increased platelet activity measured with ADP-COLL in the whole material $(\mathrm{r}=$ 0.30, $\mathrm{p}<0.05$, Fig 4a). Similar results were seen for total purines $(\mathrm{r}=-0.30, \mathrm{p}<0.05$, Fig $4 \mathrm{~b})$. There was a non-significant trend for EPI-COLL to correlate with both ADP and total purines in patients with aspirin treatment (ADP: $\mathrm{r}=-0.31, \mathrm{p}=0.09$, total purines: $\mathrm{r}=-0.32, \mathrm{p}=0.09$ ).

Aspirin resistant patients (EPI-COLL < $193 \mathrm{sec}$ ) had higher ADP levels compared to aspirin responders (734 \pm 807 vs. $282 \pm 187 \mathrm{nmol} / \mathrm{l}$, mean \pm SD, p < 0.05), and increased levels of total purines $(1615 \pm 1493$ vs. $737 \pm$ $408 \mathrm{nmol} / \mathrm{l}$, mean \pm SD, $\mathrm{p}<0.05)$.

For purine measurements there were no significant correlation with age, diabetes, haemoglobin, CKMB or troponin $\mathrm{T}$ levels. Platelet contamination was excluded by cell counting and we did not see any correlations between platelet counts and purine levels.

Nucleotide turnover is fast in whole blood due to ectonucleotidases. ATP degradation was evaluated both in samples with endogenous ATP and in samples were ATP had been added. The degradation was rapid in samples where ATP was added with a T 1/2 of $5.2 \mathrm{~min}$. Endogenous ATP levels had a slower degradation rate, with a $\mathrm{T} 1 / 2$ of approximately $30 \mathrm{~min}$.

Baseline levels of adenosine were markedly lower than those of its nucleotides and barely detectable, most likely due to both rapid uptake into the red blood cells and degradation. This was because we have not included adenosine deaminase and nucleoside transport inhibitors in the perfused solution. The magnitude of changes in adenosine levels could therefore not be studied but this was not the aim of the study.

\section{Discussion}

In agreement with previous studies we found that platelets are activated in acute coronary syndromes $[13,14]$. Furthermore, for the first time we could demonstrate a rise in systemic levels of ADP and a decreased platelet inhibitory effect of aspirin in patients with STEMI. It is possible that the raised ADP level contributes to the increased platelet activity and the reduced effect of aspirin

There is growing evidence that a significant number of patients do not benefit from therapy with standard doses of aspirin. Aspirin resistance however, is a poorly defined term describing a number of conditions including the inability of aspirin to protect individuals from cardiovascular events, to inhibit platelet aggregation measured with a number of different methods and to inhibit thromboxane
$A_{2}$ formation [15]. We found aspirin resistance levels in controls (NCD) of $9.7 \%$, which is in agreement with a previous study that found $9.5 \%$ aspirin resistance using PFA-100 [6]. It is also in agreement or even lower than other methods that found frequencies of 9-45\%. [6-8]. interestingly, we found increased frequency of aspirin resistance in acute coronary syndromes rising from $9.7 \%$ in controls to $26.0 \%$ in NSTEMI (n.s.) and $83.3 \%$ in STEMI $(p<0,001)$. In fact, we could not see any significant effect of aspirin on platelet aggregation measured with PFA-100 in acute STEMI. Thus, in the situations where the patients need the platelet inhibitory effect of aspirin the most, the aspirin resistance is most frequent

The causative factors of aspirin resistance are still unclear. Altman et al., (2004) described several possible mechanisms behind aspirin resistance and the difficulty to interpret and compare the results of different studies [16]. Both the possibilities of inadequate doses of aspirin and thromboxane independent platelet aggregation mechanisms have been discussed. In some patients aspirin resistance could simply reflect non-compliance with drug therapy. Weber et al., (2002) suggested several possible types of aspirin resistance where one is linked to inability to inhibit thromboxane formation [15]. Another possible mechanism is classified as "pseudo resistance" since aspirin exerted the expected pharmacodynamic effect of inhibiting thromboxane formation but platelet aggregation was not inhibited. Hillarp et al., (2003) found that in a series of 200 aspirin treated patients, none was found to have unblocked cyclooxygenase activity [17]. Aspirin resistance despite blocked cyclooxygenase activity has been suggested to be explained by increased platelet sensivity to ADP and collagen $[5,18]$.

Our data show that aspirin resistance increases in STEMI. This is probably due to a general activation of the platelets, because platelet aggregation was also increased in patients not treated with aspirin. The increased aspirin resistance could be explained by increased activity of important positive feedback systems such as ADP and thromboxane [19], but also by an increase in vW factor that are released from endothelial cells under high shear stress [20].

Several studies have shown sufficient inhibition of platelet aggregation with the $75 \mathrm{mg}$ dose $[1,20]$. Recently though, a randomized study of 60 patients with stable coronary artery disease showed that the effect of aspirin was dose-dependent and the conclusion was that doses of less than $100 \mathrm{mg}$ of aspirin was less effective inhibiting platelet aggregation than doses greater than $100 \mathrm{mg}$ [22]. This could indicate that higher doses of aspirin could be necessary to inhibit increased activity in the thromboxane positive feedback system, but for the time being there is 


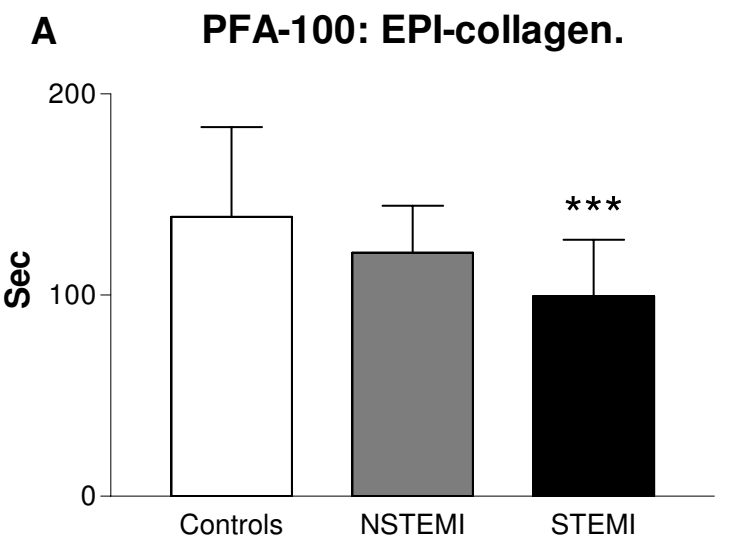

B PFA-100: ADP-collagen.

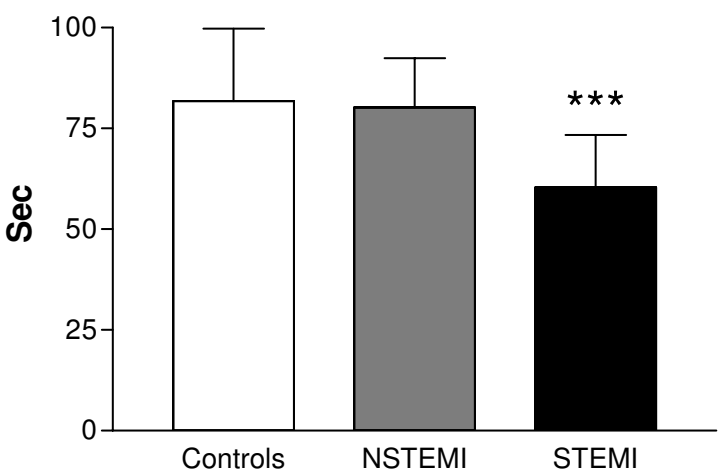

Figure I

(a) Closure time measurements (epi-collagen) in NCD (no sign of cardiac disease), NSTEMI (non ST elevation myocardial infarction) and STEMI (ST elevation myocardial infarction) groups. (b) Closure time measurements (ADP-collagen) in the NCD, NSTEMI and STEMI groups. *** $\mathrm{p}<0.001$, compared to NCD. Lower values indicate increased platelet activation.

no clear evidence saying that higher doses of aspirin improve clinical outcome [1].

However, aspirin resistance could also be dependent on increased activity of the ADP positive feedback system. $\mathrm{ADP}$ is released by activated platelets, but also in the heart from cardiac myocytes during ischemia, or from endothelial cells, red blood cells and sympathetic nerves [22,23]. Erythrocytes are known to contain large amounts of ADP, which may increase the platelet activity and modulate the effect of aspirin [24]. It is known that nucleotides are released from numerous cells during stress and exercise [23]. The control group in this study was therefore chosen

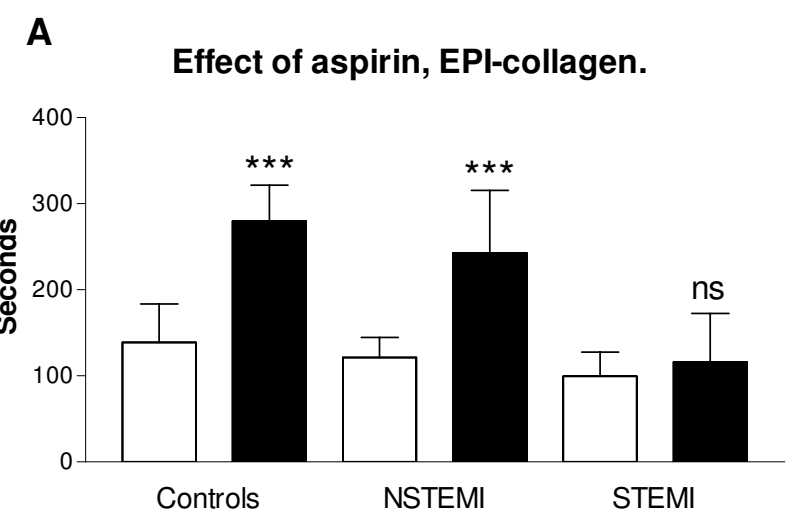

\section{B}

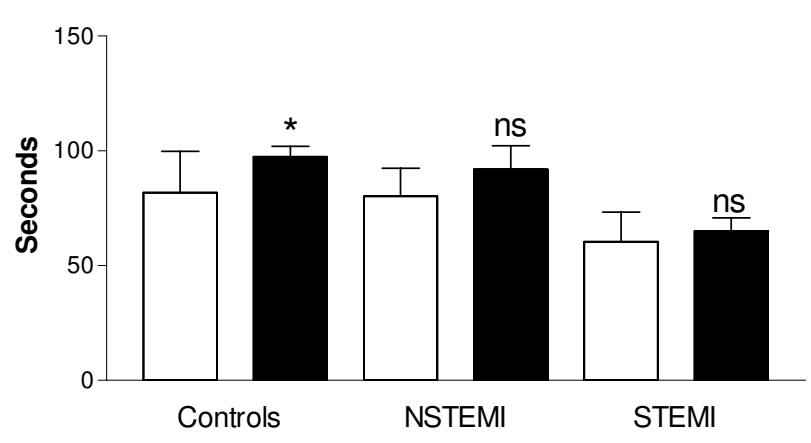

Figure 2

(a) Effect of chronic aspirin treament in the NCD (no sign of cardiac disease), NSTEMI (non ST elevation myocardial infarction) and STEMI (ST elevation myocardial infarction) groups measured as closure time with the EPI-collagen cartridge. (b) Effects of aspirin in the NCD, NSTEMI and STEMI groups measured as closure time with the ADP-collagen cartridge. White bars: no aspirin treatment, black bars: aspirin treated patients. Values are expressed as mean values $\pm S D$, $* * *$ p $<0.001$, * $\mathrm{p}<0.05$, n.s. = not significant, compared to NCD. Lower values indicate increased platelet activation.

to present pain and stress and it is our belief that this control group is more relevant for comparison than unstressed healthy individuals to prove ischemia induced increases in ADP levels.

For the first time we have demonstrated in man that ADP levels are increased during myocardial infarction. It is possible that increased ADP levels during acute myocardial ischemia could contribute to the increased frequency of aspirin resistance. There are at least two subtypes of ADP 


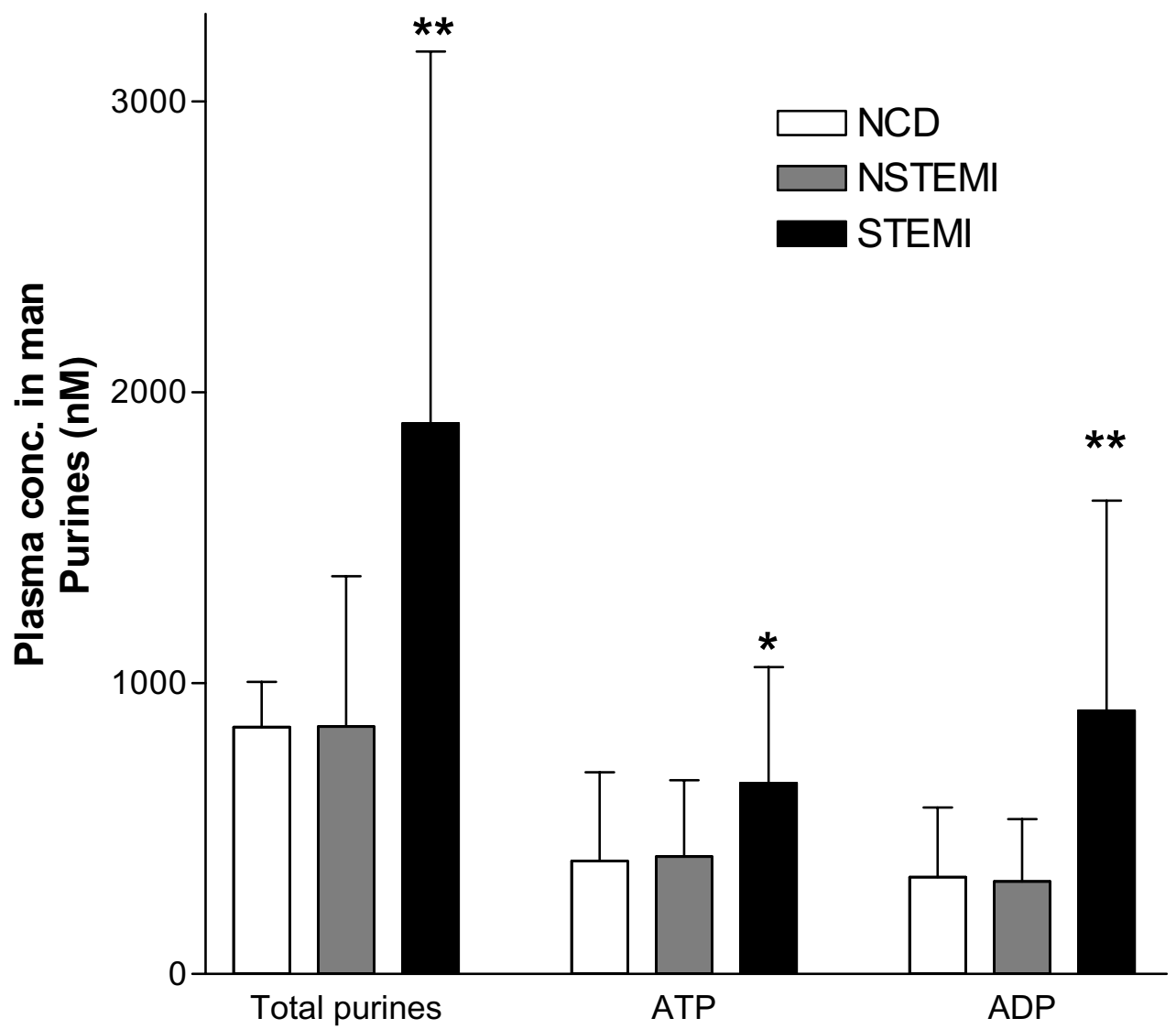

Figure 3

Plasma concentrations of extracellular purines in NCD (no sign of cardiac disease), NSTEMI (non ST elevation myocardial infarction) and STEMI (ST elevation myocardial infarction) groups expressed as mean values \pm SD. $* p<0.05$, $* * p<0.0$ I, compared to NCD.

receptors on the platelet. The $\mathrm{P} 2 \mathrm{Y}_{12}$ receptor is linked via $\mathrm{G}_{\mathrm{i}}$ protein to adenylate cyclase. The $\mathrm{P} 2 \mathrm{Y}_{12}$ receptor stimulates platelet aggregation and has a high expression in platelets $[25,26]$. Clopidogrel blocks the $\mathrm{P}_{2} \mathrm{Y}_{12}$ receptor irreversibly and the value of this treatment has been established by the CURE and CAPRIE studies. P2 $\mathrm{Y}_{1}$ is a $\mathrm{G}_{\mathrm{q}}$ protein linked ADP receptor expressed in platelets that mediates shape change $[25,26]$. P2 $\mathrm{Y}_{12}$ and thromboxane receptors act via different intracellular second messenger mechanisms, cAMP and inositol triphosphate $\left(\mathrm{IP}_{3}\right)$, respectively. This explains the additive clinical effect of clopidogrel when it is combined with aspirin $[3,4]$. However, the $\mathrm{P} 2 \mathrm{Y}_{1}$ receptor acts via the same second messenger system as thromboxane $\left(\mathrm{IP}_{3}\right)$. Thus, high levels of $\mathrm{ADP}$ could replace thromboxane as stimulator of $\mathrm{IP}_{3}$ by activation of $\mathrm{P}_{2} \mathrm{Y}_{1}$ receptors. If ADP contributes to aspirin resistance, it may not be sufficient to block the $\mathrm{P} 2 \mathrm{Y}_{12}$ receptor.
It is possible that $\mathrm{P}_{2} \mathrm{Y}_{1}$ antagonists also will be necessary to achieve inhibition of both the important intracellular second messenger systems in the platelet.

Several studies have shown a higher incidence of cardiovascular events in patients reported to be aspirin resistant. Gum et al found that aspirin resistance was associated with an increased risk of death, myocardial infarction or cerebrovascular accident compared to aspirin sensitive patients (24\% vs. $10 \%$ ) [8]. Grotemeyer and colleagues described in 1993 a 40\% risk for major events (stroke, myocardial infarction or vascular death) for aspirin resistant patients compared to a $4 \%$ risk in the aspirin responder group [7]. Eikelboom et al., (2002) recently reported that elevated urine concentrations of 11-dehydro thromboxane $\mathrm{B} 2$ could predict the risk of myocardial infarction [9]. Further prospective studies will be needed to 
A PFA-100 (ADP-coll) vs
total purine levels

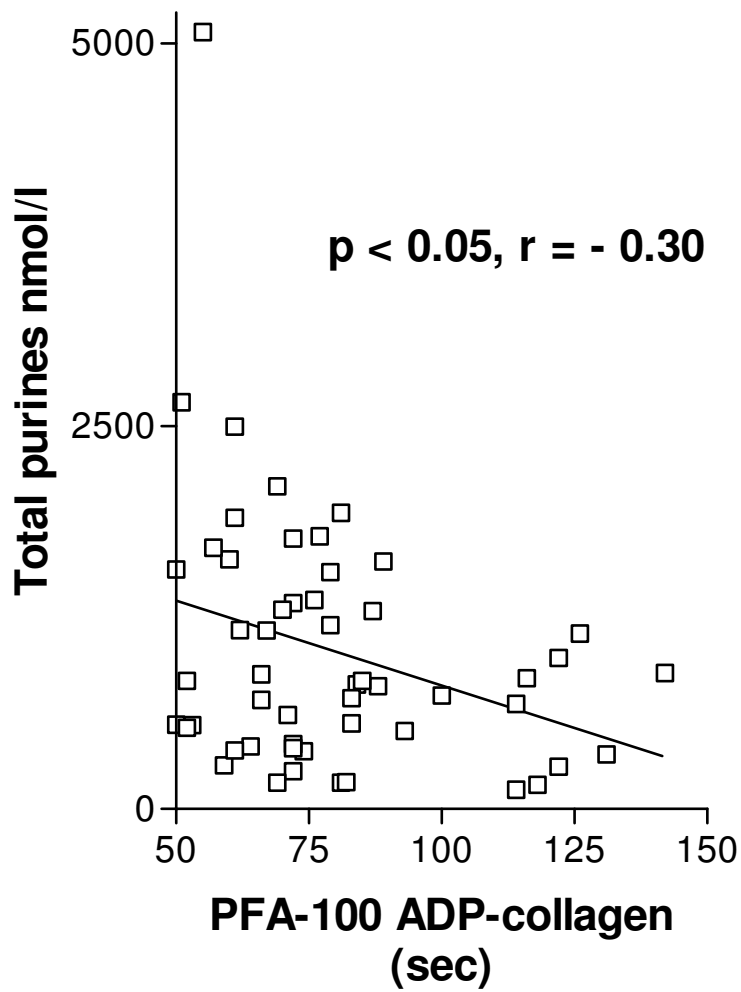

B PFA-100 (ADP-coll) vs ADP levels

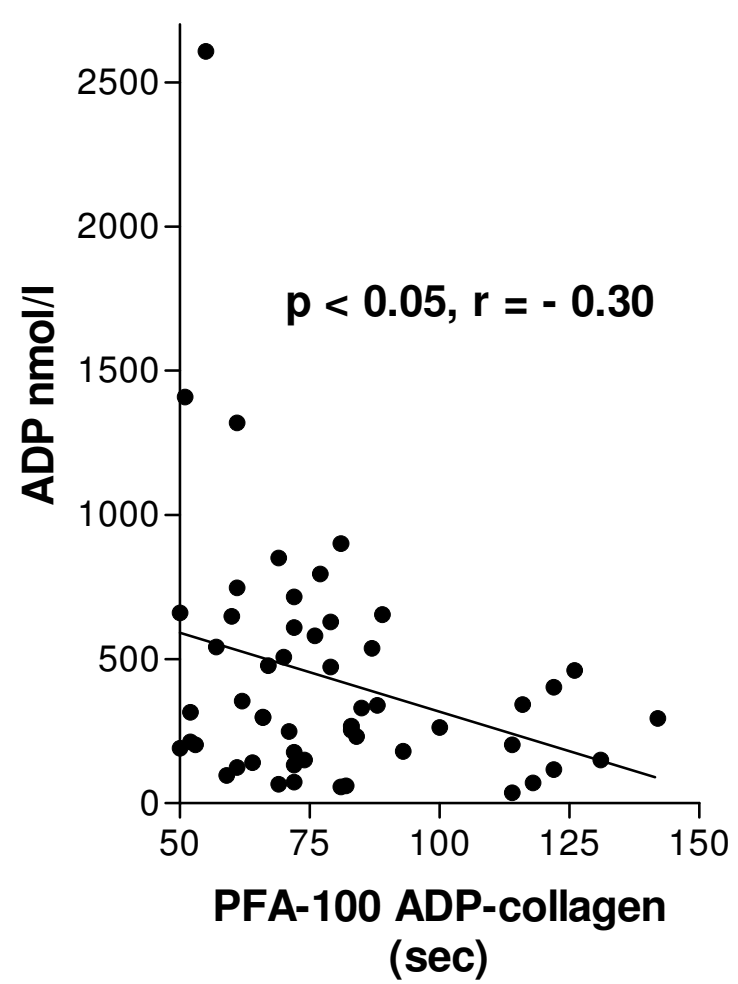

Figure 4

(a) Correlation between extracellular total purine levels and platelet activity measured as closure time with the ADP-COLL cartridge. (b) Correlation between extracellular ADP levels and platelet activity measured as closure time with the ADP-COLL cartridge.

evaluate if ADP levels could be of importance in the identification of patients with increased risk of myocardial infarction in the future. Why did the patients with aspirin resistance have higher ADP levels? It is possible that this was the result of a more pronounced cardiac ischemia. However, ADP levels did not correlate with either CKMB or troponin T levels. Another explanation could be that subgroups of patients are more dependent on the ADP positive feedback system than on the thromboxane system, and therefore more aspirin resistant. Then our finding of increased ADP levels may reflect an increased ADP release from platelets. These patents may benefit more from inhibitors of ADP mediated platelet activation than aspirin.

\section{Limitations of the study}

Patients found to be aspirin resistant were not all confirmed by a second test with the PFA-100. However, this was not possible in the NSTEMI and STEMI groups since they were treated early with clopidogrel, enoxaparin or GPIIb/IIIa-blockers that influence the PFA-100 measurements. ADP measurements are difficult because of the rapid degradation by ectonucleotidases being present predominantly on endothelial cells. We found a half-life of 5.2 min when ATP was added to our blood samples in vitro, however endogenous levels of ATP levels were more stable. The baseline ATP levels were in the micromolar range, which is similar to previously reported levels of circulating ATP in man [27]. Our sampling of venous blood in the antecubital vein is clearly not optimal to detect purine release in the heart. The released purine has passed both the lung and systemic circulation resulting in a degradation chain from ATP, ADP, AMP to adenosine. Adenosine is then rapidly taking up by the red blood cells. Thus the adenosine levels were barely detectable. It is our belief that blood sampling directly from heart veins 
Table I: The clinical characteristics of the NCD (no sign of cardiac disease), NSTEMI (non ST elevation myocardial infarction) and STEMI (ST elevation myocardial infarction) groups. Values are expressed as mean \pm SD or numbers.

\begin{tabular}{lccc}
\hline Characteristics & Controls $n=67$ & NSTEMI $n=38$ & STEMI $n=30$ \\
\hline Age & $66 \pm 12$ & $72 \pm 15$ & $72 \pm 13$ \\
M/F & $46 / 21$ & $29 / 9$ & $21 / 9$ \\
Diabetes Mellitus & 8 & 8 & 4 \\
Prior IHD & 29 & 18 & 12 \\
Hemoglobine, $g / l$ & $135 \pm 15$ & $132 \pm 15$ & $132 \pm 16$ \\
Platelet count, $\times 109 / /$ & $224 \pm 74$ & $218 \pm 51$ & $222 \pm 66$ \\
Cholesterol mmol/l & - & $4,67 \pm 0,94$ & $4,60 \pm 0,79$ \\
Triglycerides mmol/l & - & $1,19 \pm 0,46$ & $1,28 \pm 0,94$ \\
BMI & - & $24 \pm 2,8$ & $27 \pm 3,2$ \\
\hline
\end{tabular}

would have resulted in markedly increased purine levels in the NSTEMI and STEMI groups and probably also better correlations with platelet activity. Another limitation is that the correlation analysis had to be done on the whole material since the subgroups were too small for separate correlation analysis.

\section{Conclusion}

Platelets are activated and aspirin resistance is more frequent in STEMI, probably due to a general activation of platelets. ADP levels are increased in STEMI and correlates with platelet activation. Increased levels of ADP could be one reason for increased platelet activity and aspirin resistance.

\section{Authors' contributions}

$\mathrm{CB}$ was the principal investigator for the study, responsible for recruitment of patients, aspirin resistance analysis, study design and wrote the manuscript.

$\mathrm{CvH}$ performed the purine analytical assays.

EL performed the purine analytical assays and participated in writing the manuscript.

HÖ participated in study design, helped in recruiting patients and wrote the manuscript.

DE conceived the study, guided throughout the study and wrote the manuscript.

All authors read and approved the final manuscript.

\section{Acknowledgements}

The study has been supported by the Swedish Heart and Lung Foundation, the Franke and Margareta Bergqvist Foundation, the Wiberg Foundation, the Bergwall Foundation, the Zoegas Foundation, the Westergren Foundation, the Swedish Medical Society, and the Swedish Medical Research Council, Grant I3|30.

\section{References}

I. Antithrombotic Trialists' Collaboration: Collaborative meta-analysis of randomised trials of antiplatelet therapy for prevention of death, myocardial infarction, and stroke in high risk patients. BM/ 2002, 324:7I-86.

2. CAPRIE Steering Committee: A randomised, blinded, trial of clopidogrel versus aspirin in patients at risk of ischaemic events (CAPRIE). Lancet 1996, 348: I329-39.

3. The Clopidogrel in Unstable angina to prevent Recurrent Events (CURE) trial investigators: Effects of clopidogrel in addition to aspirin in patients with acute coronary syndromes without ST-segment elevation. New Engl J Med 2001, 345:494-502.

4. Steinhubl SR, Berger PB, Mann JT 3rd, Fry ET, DeLago A, Wilmer C, Topol Ej: Early and sustained dual oral antiplatelet therapy following percutaneous coronary intervention: a randomized controlled trial. JAMA 2002, 288:24II-20.

5. Macchi L, Christiaens L, Brabant S, Sorel N, Allal J, Mauco G, Brizard $A$ : Resistance to aspirin in vitro is associated with increased platelet sensitivity to adenosine diphosphate. Thromb Res 2002, 107:45-49.

6. Gum PA, Kottke-Marchant K, Poggio ED, Gurm H, Welsh PA, Brooks L, Sapp SK, Topol EJ: Profile and prevalence of aspirin resistance in patients with cardiovascular disease. Am J Cardiol $200 \mathrm{I}$, 88:230-235.

7. Grotemeyer KH, Scharafinski HW, Husstedt IW: Two-year followup of aspirin responder and aspirin non responder. A pilotstudy including 180 post-stroke patients. Thromb Res 1993 , 71:397-403.

8. Gum PA, Kottke-Marchant K, Welsh PA, White J, Topol E: A prospective, blinded determination of the natural history of aspirin resistance among stable patients with cardiovascular disease. J Am Coll Cardiol 2003, 4I:96I-965.

9. Eikelboom JW, Hirsh J, Weitz JI, Johnston M, Yi Q, Yusuf S: Aspirinresistant thromboxane biosynthesis and the risk of myocardial infarction, stroke, or cardiovascular death in patients at high risk for cardiovascular events. Circulation 2002, 105:1650-5.

10. Kundu SK, Heilmann EJ, Sio R, Garcia C, Davidson RM, Ostgaard RA: Description of an in vitro platelet function analyzer-PFA100. Semin Thromb Hemost 1995, 21 (Suppl 2): 106-12.

II. Lazarowski ER, Boucher RC, Harden TK: Constitutive release of ATP and evidence for major contribution of ecto-nucleotide pyrophosphatase and nucleoside diphosphokinase to extracellular nucleotide concentrations. I Biol Chem 2000, 275:3106I-31068.

12. Levitt B, Head RJ, Westfall DP: High-pressure liquid chromatographic fluorometric detection of adenosine and adenine nucleotides: application to endogenous content and electrically induced release of adenyl purines in guinea pig vas deferens. Anal Biochem 1984, 137:93-100.

13. Kristensen SD, Bath PM, Martin JF: Differences in bleeding time, aspirin sensitivity and adrenaline between acute myocardial infarction and unstable angina. Cardiovasc Res 1990, 24:19-23. 
14. Milner PC, Martin JF: Shortened bleeding time in acute myocardial infarction and its relation to platelet mass. $\mathrm{Br} \mathrm{Med} \mathrm{J} \mathrm{(Clin}$ Res Ed) 1985, 290: 1767-70.

15. Weber AA, Przytulski B, Schanz A, Hohlfeld T, Schror K: Towards a definition of aspirin resistance: a typological approach. Platelets 2002, I:37-40.

16. Altman R, Luciardi HL, Muntaner J, Herrera RN: The antithrombotic profile of aspirin. Aspirin resistance, or simply failure? Thromb J 2004, 2:I.

17. Hillarp A, Lethagen S, Mattiasson I: Aspirin resistance is not a common biochemical phenotype explained by unblocked cyclooxygenase-I activity. I Thromb Haemost 2003, I: 196-197.

18. Kawasaki T, Ozeki Y, Igawa T, Kambayashi J: Increased platelet sensitivity to collagen in individuals resistant to low-dose aspirin. Stroke 2000, 31:591-5.

19. Rasmanis G, Vesterqvist O, Green K, Edhag O, Henriksson P: Effects of intermittent treatment with aspirin on thromboxane and prostacyclin formation in patients with acute myocardial infarction. Lancet 1988, 2(8605):245-7.

20. Heper G, Bayraktaroglu M: The importance of von Willebrand factor level and heart rate changes in acute coronary syndromes: a comparison with chronic ischemic conditions. Angiology 2003, 54:287-99.

21. Buerke M, Pittroff W, Meyer J, Darius H: Aspirin therapy: optimized platelet inhibition with different loading and maintenance doses. Am Heart J 1995, I 30(3 Pt I):465-72.

22. Malhotra S, Sharma YP, Grover A, Majumdar S, Hanif SM, Bhargava VK, Bhatnagar A, Pandhi P: Effect of different aspirin doses on platelet aggregation in patients with stable coronary artery disease. Intern Med J 2003, 33:350-4.

23. Gordon JL: Extracellular ATP: effects, sources and fate. Biochem J 1986, 233:309-19.

24. Vassort G: Adenosine 5'-Triphosphate: a P2-purinergic agonist in the myocardium. Physiological Reviews 2001:767-806.

25. Valles J, Santos MT, Aznar J, Osa A, Lago A, Cosin J, Sanchez E, Broekman MJ, Marcus AJ: Erythrocyte promotion of platelet reactivity decreases the effectiveness of aspirin as an antithrombotic therapeutic modality: the effect of low-dose aspirin is less than optimal in patients with vascular disease due to prothrombotic effects of erythrocytes on platelet reactivity. Circulation 1998, 97:350-355.

26. Storey RF, Newby LJ, Heptinstall S: Effects of P2Y(I) and P2Y(I 2) receptor antagonists on platelet aggregation induced by different agonists in human whole blood. Platelets 200 I, I 2:443-7.

27. Wang L, Östberg O, Wihlborg A-K, Brogren $H$, Jern S, Erlinge D: Quantification of ADP and ATP Receptor Expression in Human Platelets. J Thromb \& Haem 2003, I:330-336.

28. González-Alonso J, Olsen DB, Saltin B: Erythrocyte and the regulation of human skeletal muscle blood flow and oxygen delivery. Role of circulating ATP. Circ Res 2002, 91:1046-1055.
Publish with Biomed Central and every scientist can read your work free of charge

"BioMed Central will be the most significant development for disseminating the results of biomedical research in our lifetime. "

Sir Paul Nurse, Cancer Research UK

Your research papers will be:

- available free of charge to the entire biomedical community

- peer reviewed and published immediately upon acceptance

- cited in PubMed and archived on PubMed Central

- yours - you keep the copyright
BioMedcentral 